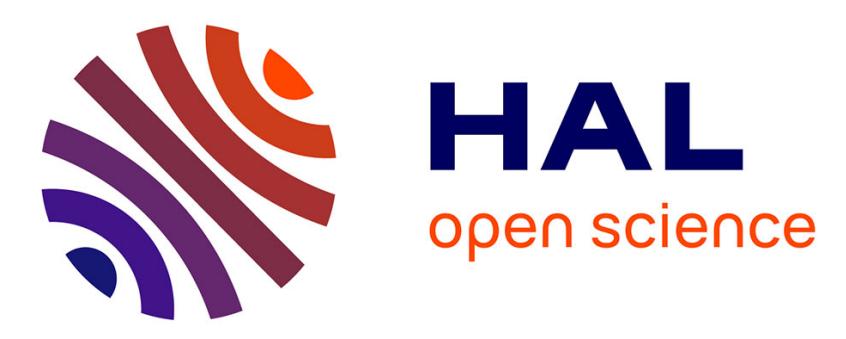

\title{
Three-dimensional trapping of light with light in semiconductor planar microcavities
}

S. Anguiano, A. A Reynoso, A. Bruchhausen, Aristide Lemaitre, J. Bloch, A. Fainstein

\section{- To cite this version:}

S. Anguiano, A. A Reynoso, A. Bruchhausen, Aristide Lemaitre, J. Bloch, et al.. Three-dimensional trapping of light with light in semiconductor planar microcavities. Physical Review B: Condensed Matter and Materials Physics (1998-2015), 2019, 99 (19), 10.1103/PhysRevB.99.195308 . hal-02352310

\section{HAL Id: hal-02352310 https://hal.science/hal-02352310}

Submitted on 6 Nov 2019

HAL is a multi-disciplinary open access archive for the deposit and dissemination of scientific research documents, whether they are published or not. The documents may come from teaching and research institutions in France or abroad, or from public or private research centers.
L'archive ouverte pluridisciplinaire HAL, est destinée au dépôt et à la diffusion de documents scientifiques de niveau recherche, publiés ou non, émanant des établissements d'enseignement et de recherche français ou étrangers, des laboratoires publics ou privés. 


\title{
Three-dimensional trapping of light with light in semiconductor planar microcavities
}

\author{
S. Anguiano, ${ }^{1}$ A. A. Reynoso, ${ }^{1}$ A. E. Bruchhausen, ${ }^{1}$ A. Lemaître, ${ }^{2}$ J. Bloch,${ }^{3}$ and A. Fainstein ${ }^{1, *}$ \\ ${ }^{1}$ Centro Atómico Bariloche and Instituto Balseiro, CNEA, CONICET, 8400 San Carlos de Bariloche, Río Negro, Argentina \\ ${ }^{2}$ Centre de Nanosciences et de Nanotechnologies, CNRS, Univ. Paris-Sud, Universiteé Paris-Saclay, 91120 Palaiseau, France \\ ${ }^{3}$ Centre de Nanosciences et de Nanotechnologies (C2N), Avenue de la Vauve, 911200 Palaiseau, France
}

(Received 7 December 2018; revised manuscript received 13 May 2019; published 29 May 2019)

\begin{abstract}
When light is confined in all three directions and in dimensions of the order of the light wavelength, discretization of the photon spectra and distinctive phenomena occur, the Purcell effect and the inhibition of emission of atoms being two paradigmatic examples. Diverse solid-state devices that confine light in all three dimensions have been developed and applied. Typically the confinement volume, operating wavelength, and quality factor of these resonators are set by construction, and small variations of these characteristics with external perturbations are targeted for applications including light modulation and control. Here we describe full three-dimensional light trapping, that is set and tuned by laser excitation in an all-optical scheme. The proposed device is based on a planar distributed Bragg reflector GaAs semiconductor microcavity operated at room temperature. Lateral confinement is generated by an in-plane gradient in the refractive index of the structure's materials due to localized heating, which is in turn induced by carriers photoexcited by a focused laser. Strong three-dimensional trapping of light is evidenced by the laser-induced changes on the spectral, spatial, and $k$-space distribution of the emission. The dynamics of the laser-induced photonic potential is studied using modulated optical excitation, highlighting the central role of thermal effects at the origin of the observed phenomena.
\end{abstract}

DOI: 10.1103/PhysRevB.99.195308

\section{INTRODUCTION}

Resonant cavities are pervasive to many different natural phenomena and man-made devices. Standing waves confined between reflective walls build up, localizing the wave energy and enhancing their interaction with other physical degrees of freedom. They are ubiquitous in the domain of optics, for example as the basic feedback mechanism of lasers [1,2], or in the most sensitive displacement sensors, such as the Fabry-Perot cavities used for the detection of gravitational waves at LIGO [3]. When the confinement is strong and in all three dimensions, a discretization of part of the photon spectra can occur $[2,4-6]$, leading to distinctive phenomena such as, for example, the inhibition or enhancement of the emission of atoms [7-9], and the Bose-Einstein condensation of strongly coupled matter and light particles (cavity polaritons) $[6,10]$. In the NIR-visible spectral range, such three-dimensional photon trapping typically requires the development of confining structures based on nano- and microfabrication methods. Diverse techniques have been developed and applied, including the use of local defects in periodic photonic band gap freestanding membranes [11], the introduction of traps in planar distributed Bragg reflector (DBR) cavities through defects such as GaAs droplets [12] or lateral patterning [2,6,13], or their full three-dimensional (3D) structuring into microdisks and pillar resonators based on finely tuned ion etching methods $[4,5,14]$. These techniques are already mature and robust, leading to high-quality devices that have had a huge impact on

\footnotetext{
${ }^{*}$ Correspondence and requests for materials should be addressed to afains@cab.cnea.gov.ar
}

the study of novel optical, optoelectronic, and optomechanical phenomena in the last couple of decades. One key targeted application has been that of all-optical modulation and control of light. Being solid-state devices, however, their flexibility regarding their confinement characteristics and operating wavelength is limited, since it is typically defined by design and construction. In the most standard approach, highly confining resonators are used with the ultranarrow cavity mode subtly tuned by small changes in refractive index $[15,16]$. A different situation would be attained if light trapping could not only be tuned but also set in an all-optical scheme. Such tunable 3D trapping of light is what we describe here.

Our approach to this task is based on a planar DBR semiconductor microcavity for the light confinement in one direction $(z)$, plus a photon potential defined by a focused laser for the in-plane additional trapping [see the scheme in Fig. 1(a)]. When a semiconductor is optically excited above or close to an electronic transition, a change of the refractive index occurs. This can be due to the modification of the electronic bands or the accessible electronic states induced by the presence of excited carriers $[17,18]$, or by the lattice heating produced by the relaxation of these carriers [19]. If the change is positive, the local value of the cavity mode energy will be decreased and then light trapping will be possible. A conceptual scheme of the so-obtained 3D effective attractive photon potential is shown in Fig. 1(b).

In the transparency region of a semiconductor, and for a fixed wavelength, the refractive index is expected to increase if the band gap is reduced. A gap reduction could be produced by an increase of the local temperature generated by photocarrier-induced laser heating. However, more complex nonlinearities related to photoexcited carrier correlations 

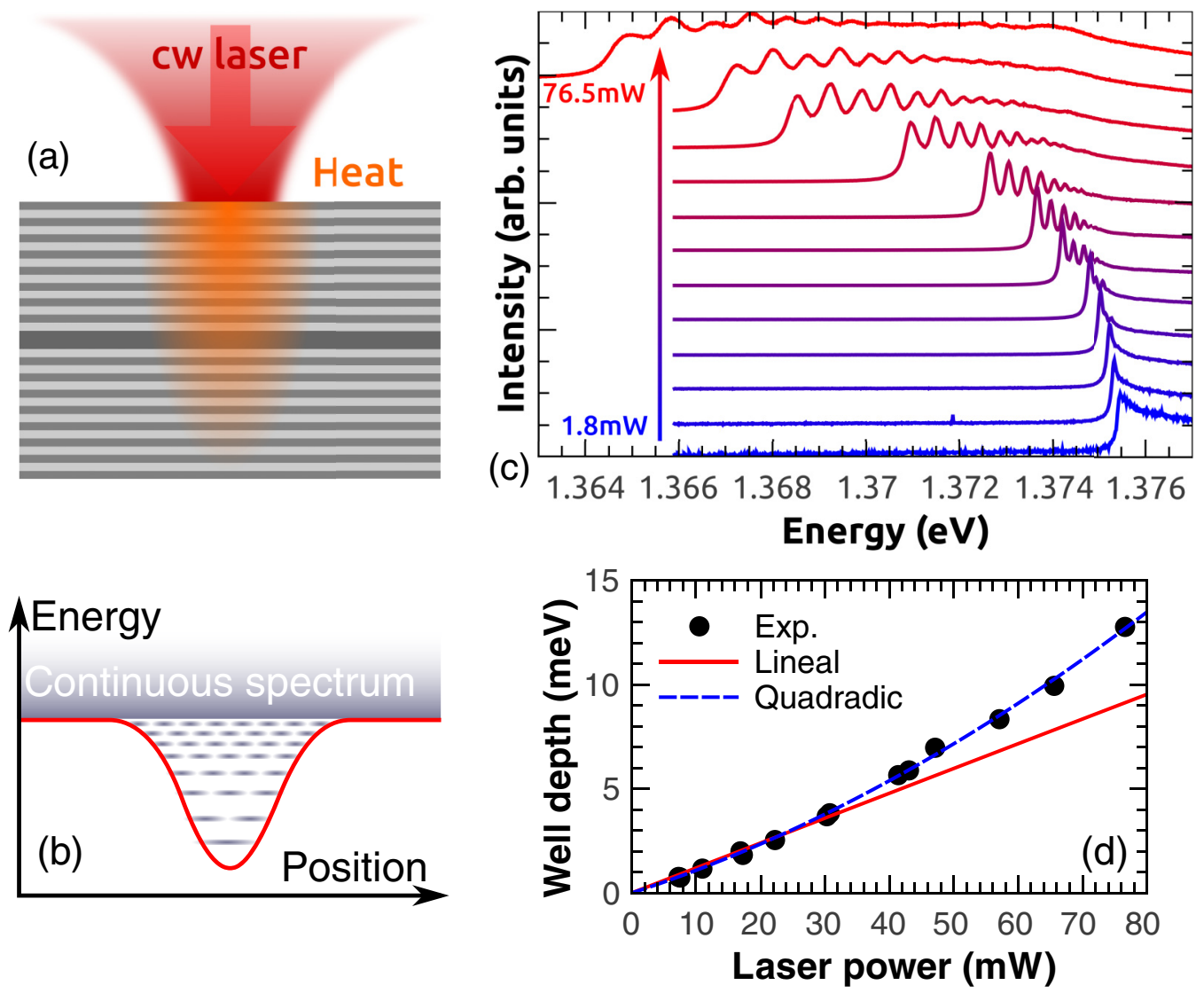

FIG. 1. (a) Sketch of the system, showing the generation of electron-hole pairs and the resulting temperature distribution due to their relaxation with the lattice. (b) Representation of a Gaussian optical well and the resulting confined modes, which appear beneath the original continuous spectrum. (c) Photoluminescence spectra as a function of incident power, ranging from 1.8 up to $76.5 \mathrm{~mW}$. (d) Optical potential well depth as a function of laser power. The black circles are experimental results, while the red line is a linear fit to the low power ( $\leqslant 30 \mathrm{~mW}$ ) part of the data, and the blue dashed line is a quadratic fit to the whole data.

could in principle also be involved [17]. For example, polariton energy barriers (in contrast with traps) have been demonstrated as a result of the blueshift of the polariton branch due to polariton-polariton interactions in microcavities at low temperatures and in the strong-coupling regime [20-22]. Trapping can be induced, for example, by means of potential barriers generated with ringlike laser illumination, as reported for cold excitons in indirect quantum wells [23]. Optically generated local traps in planar microcavities with attractive weak potentials have been shown to stabilize polariton condensates with a mechanism based on spatially confined gain [24], similar to gain guiding in semiconductor laser structures [25]. Also, positive changes of the refractive index have been reported to affect photon lasing in GaAs microcavities when evolving from the excitonic regime toward the electron-hole plasma regime with increasing photoexcitation power [26]. In contrast with these low-temperature carrier-related correlation effects, we will demonstrate that strong three-dimensional trapping of photons can be achieved in planar semiconductor microcavities at room temperature through photothermal phenomena.

\section{SAMPLE AND EXPERIMENTAL SET-UP}

The studied sample consists of a high $Q$-factor $\lambda / 2$ bulkGaAs planar cavity enclosed by two DBRs consisting of alternating $\mathrm{Ga}_{0.9} \mathrm{Al}_{0.1} \mathrm{As} / \mathrm{Ga}_{0.05} \mathrm{Al}_{0.95} \mathrm{As} \lambda / 4$ layers, 28 pairs on the bottom, and 24 on top. The structure was grown by molecular beam epitaxy on an $\sim 400-\mu$ m-thick GaAs substrate. The structure presents a gradient in the layers' thicknesses that allows one to tune the optical mode around the GaAs electronic transition at $\sim 1.42 \mathrm{eV}$ just by shifting the position of the laser spot on the sample. The reported experiments correspond to room-temperature photoluminescence microscopy measurements, with the optical cavity mode tuned below the GaAs gap. In high-purity devices like the ones studied in this work the emission at energies below the electronic resonance is known to be mostly due to phonon-assisted recombination [27-29].

The optical setup, described in the Supplemental Material [30], allows for the acquisition of the integrated emitted spectra, or alternatively the spatial or $k$-dispersion profile of the emission [31]. The structure was fixed with heat-conducting silver paint on a copper holder in equilibrium with ambient temperature, and was excited with a $1.63 \mathrm{eV}$ continuous-wave (cw) Ti:sapphire laser. A $20 \times$ objective lens with a numerical aperture of 0.3 was used for both excitation and light collection, giving a Gaussian spot with full width at half maximum (FWHM) of $\sim 10 \mu \mathrm{m}$. Figure 1(c) presents a few examples of the photoluminescence spectra obtained when exciting with different incident powers, with the optical cavity mode tuned 

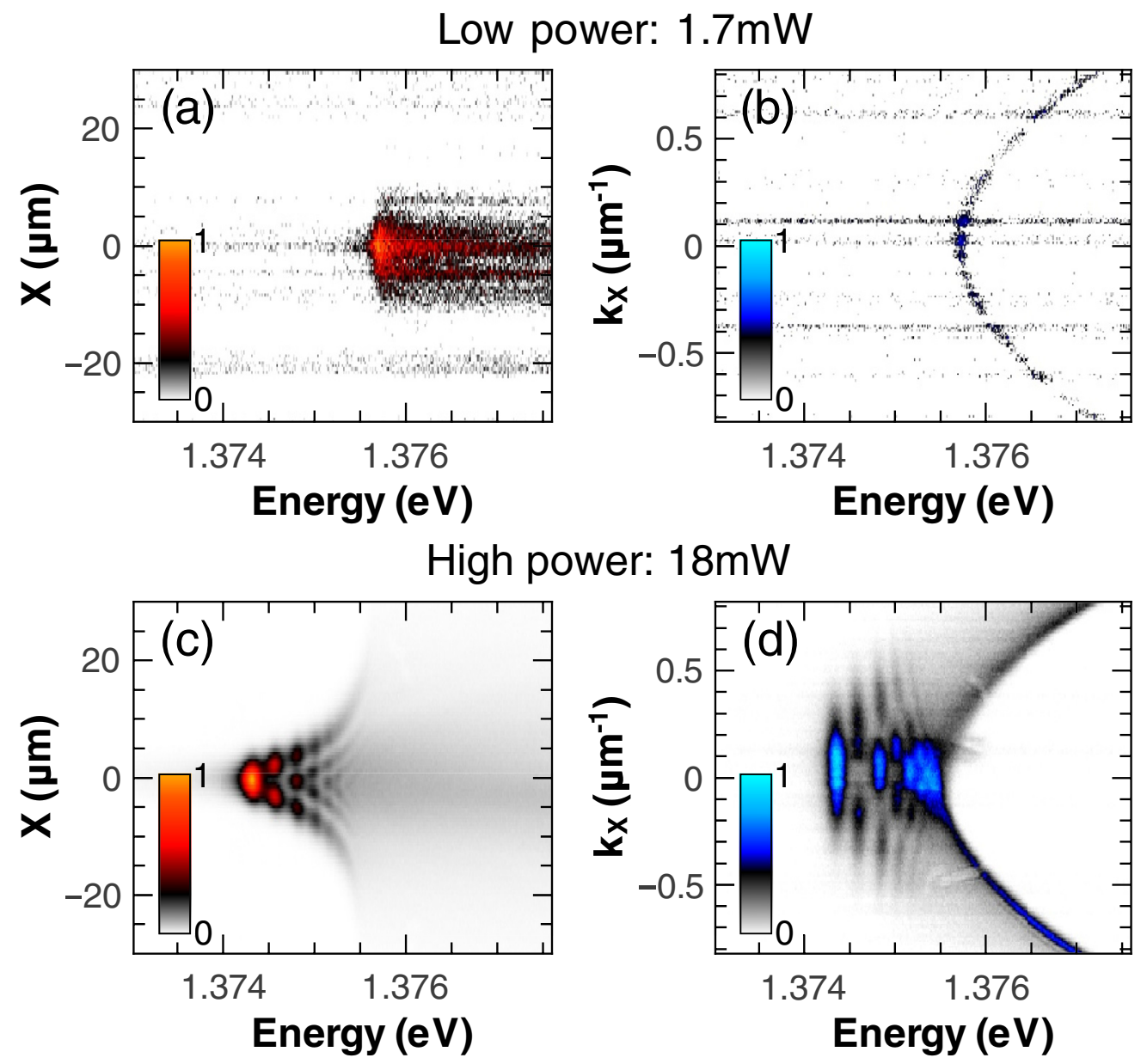

wer: $18 \mathrm{~mW}$

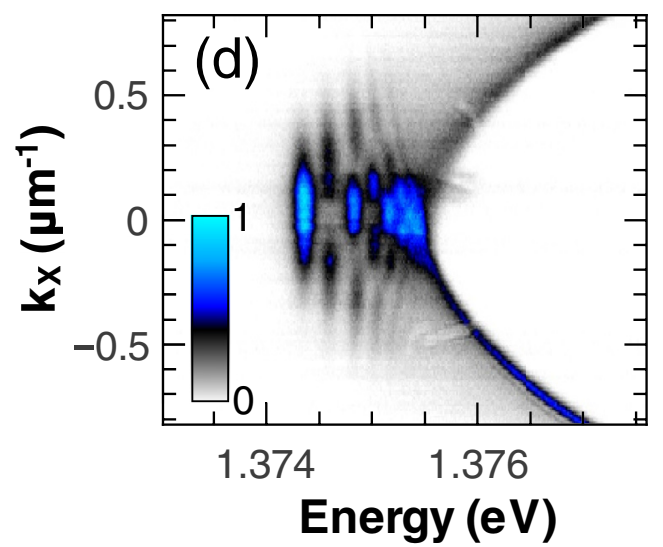

FIG. 2. Photoluminescence spectra spatial [(a),(c)] and angular [(b),(d)] resolution. The colors chosen for the spatial (orange) and the angular (blue) images are different to help in their rapid identification. The sample was excited with $\sim 1.7 \mathrm{~mW}[(\mathrm{a})$, (b)] and $\sim 18 \mathrm{~mW}$ $[(\mathrm{c}),(\mathrm{d})]$.

below but not far from the GaAs gap $\left(\varepsilon_{\text {gap }} \sim 1.42 \mathrm{eV}\right.$ at room temperature), and collected over the full angular range of the objective (equivalent to $\mathrm{k}_{r} \approx 2.1 \mu \mathrm{m}^{-1}$ ).

\section{RESULTS AND DISCUSSION}

The low power spectrum in Fig. 1(c) $(1.8 \mathrm{~mW})$ is representative of the planar microcavity [one-dimensional (1D) confinement] situation. The planar cavity quality factor in the transparency region, below the GaAs gap, is around $1 \times 10^{4}$. This corresponds, for the emission normal to the structure, to a narrow peak of $\sim 150 \mu \mathrm{eV}$ width centered in this case at $\sim 1.3755 \mathrm{eV}$. The confined cavity mode emission along $z$ defines the low-energy flank of this curve. Its flat continuation toward higher energies is determined by the in-plane parabolic dispersion of the planar cavity optical mode, which is collected within the numerical aperture of the microscope objective [32]. Major and quite striking changes in the spectra occur when the focused laser power is increased. Some conspicuous new features arise, namely, a redshift of the emission, and the appearance of clear peaks contrasting with the essentially featureless low-power spectrum. As we argue next, these features evidence the optical confinement within a Gaussian photon potential well, which is induced by the local heating of the sample due to the laser excitation. The trapping energy can attain quite significant values under the focusing conditions used, e.g., $\sim 13 \mathrm{meV}$ for a $\sim 76.5 \mathrm{~mW}$ excitation, a value equivalent to 100 times the FWHM of the unperturbed planar cavity mode.

Three-dimensional confinement of light should be reflected in the induced changes of the spectra [as manifested by the energy lowering and spectral discretization in Fig. 1(c)], but also by the corresponding modified spatial and $k$-space (angular) characteristics of the photoluminescence emission [33]. Figure 2 presents the spatial [(a),(c)] and angular [(b),(d)] distributions of the emitted light when a low- [(a),(b)] or high[(c),(d)] power excitation is applied. Again, the low-power panels $(1.7 \mathrm{~mW})$ are representative of the planar (1D confinement) case. As expected, the spatial distribution reproduces the shape of the excitation Gaussian profile. Its in-plane $k$ dispersion in turn reflects the typical parabolic behavior of light confined in one dimension, but free to propagate in the other two (in-plane) directions. The situation dramatically changes when increasing the focused laser power (18 mW in Fig. 2). New discretized modes appear at lower energies, with spatial and in-plane $k$ dispersion strongly resemblant of laterally confined modes, as seen, e.g., in micropillar cavities $[4,5,34]$ or in laterally microstructured planar resonators $[6,35]$. The spatial 

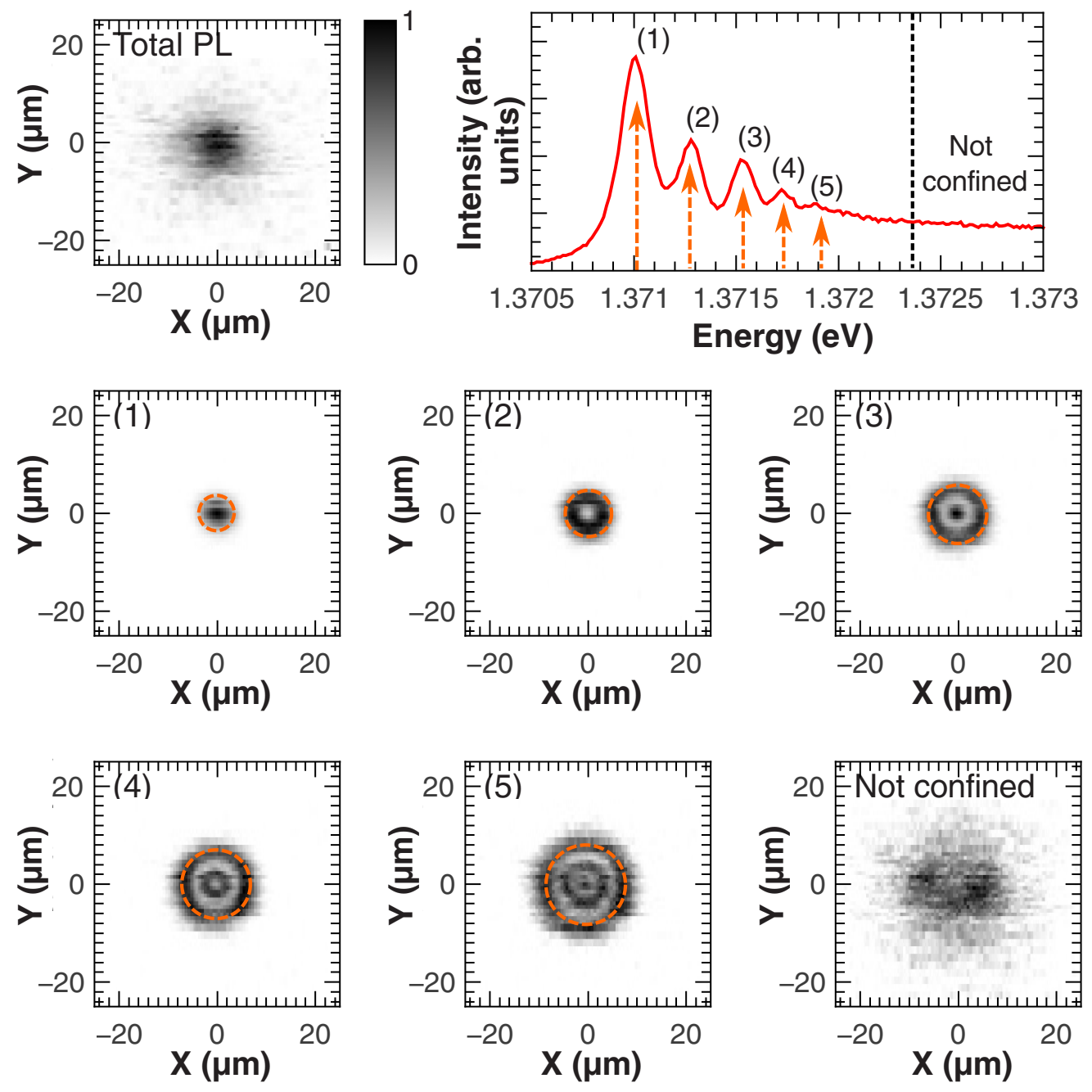

FIG. 3. Spatial distribution of the photoluminescence spectra for an excitation of $\sim 18 \mathrm{~mW}$. The top-leftmost graph corresponds to the reconstructed spatial image of the emitted photoluminescence. The red curve in the top-rightmost graph is the spectral distribution of the emitted light coming from the central line $(Y=0 \mu \mathrm{m})$ of the spot. The rest are different energy cuts [with increasing energy from left to right and top to bottom, labeled from (1) to (5)]. The dashed orange arrows and circles show the calculated energies and effective diameters of the confined modes, respectively. The vertical dashed line in the top-rightmost panel corresponds to $\varepsilon_{\infty}$.

and spectral envelope of the observed modes in Fig. 2(c) nicely reflects the Gaussian photon potential trap induced by the focused laser.

In Fig. 3 we show the lateral distribution of the emitted light for $18 \mathrm{~mW}$ excitation. The top-left graph corresponds to the total integrated emission, reflecting the homogeneous spatial distribution of the excitation beam. The spectrum displayed in the top-right panel corresponds to this emission when the spot is imaged centered on the spectrometer entrance slit. The vertical dotted line in this latter panel shows the energy of the unperturbed cavity mode, above which modes are not confined. The discrete peaks (1)-(5) correspond to the observed fully 3D confined photonic states. The six remaining graphs in this figure are constant energy sections that were selected to leave only one mode for each cut [labeled with the same numbers (1)-(5)], and the nonconfined spectral region. Circularly symmetric modes confined within different radial distances are clearly identified.
The spectral and spatial distributions of the observed modes have the required information to model the laserinduced photon potential. Regardless of the exact physical process, which we will approach later on, it is apparent that the laser excitation is generating a local change in the optical properties of the structure, which in turn produces an optical potential well that confines light in an analogous way as that observed in a micropillar structure [4]. Based on the excitation profile and symmetry of the studied system, a Gaussian well of finite depth is expected to be generated:

$$
\varepsilon=\varepsilon_{\infty}-\Delta \varepsilon_{0} e^{-r^{2} / 2 \sigma^{2}}
$$

where $r$ is the radius, $\sigma$ the standard deviation, $\varepsilon_{\infty}$ the energy of the continuum (datum obtained from the experiment), and $\Delta \varepsilon_{0}$ the effective depth of the well. The problem can be significantly simplified by making the approximation that each confined mode of energy $\varepsilon$, interacts with an infinitely deep circular well of diameter $D_{\text {eff }}$, given by (1). This is equivalent 


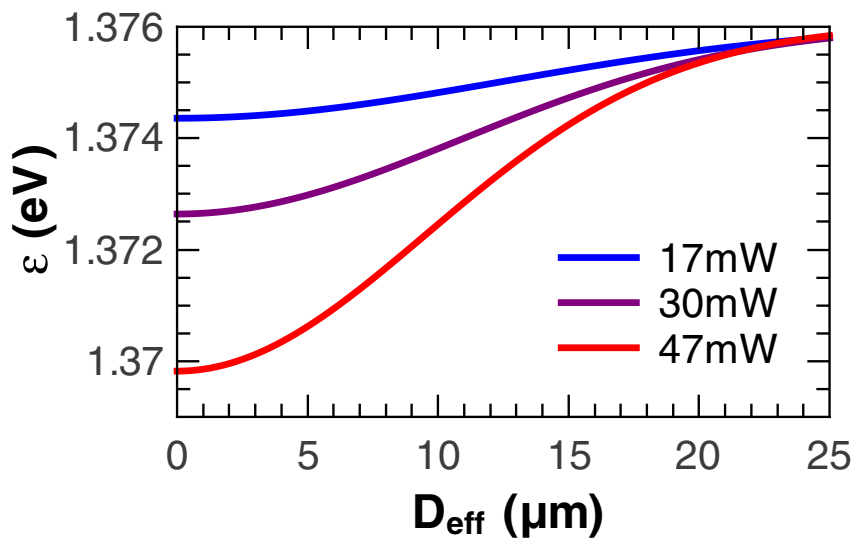

FIG. 4. Comparison between the obtained optical potential wells for three different excitation powers.

to considering that the confined modes present a node on the wall of the Gaussian well, if we define $D_{\text {eff }}$ so that it follows the Gaussian shape of the actual potential well. Therefore, reformulating Eq. (1), we get the dependence between the mode energy and the effective diameter of the infinitely deep well that best approximates the problem:

$$
\varepsilon=\varepsilon_{\infty}-\Delta \varepsilon_{0} e^{-D_{\mathrm{eff}}^{2} / 8 \sigma^{2}}
$$

being $\Delta \varepsilon_{0}=\varepsilon_{\infty}-\varepsilon_{0}$ the potential well depth and $\varepsilon_{0}$ the energy of the bottom of the potential well.

Under these assumptions, the confined modes can be well represented with Bessel functions, and their energies can be calculated as [33]

$$
\varepsilon=\sqrt{\varepsilon_{0}^{2}+\frac{4 \hbar^{2} c^{2}}{n_{c a v}{ }^{2}} \frac{X_{m l}^{2}}{D_{\mathrm{eff}}^{2}},}
$$

where $X_{m l}$ is the $m$ th root of the Bessel function of order 1, $c$ the speed of light in vacuum, and $n_{\text {cav }}$ the effective index of refraction of the sample. Equations (2) and (3) can be combined to obtain $\varepsilon_{0}$ and $\sigma$ as fitting parameters, with the measured energies $\varepsilon$ and the corresponding Bessel roots $X_{m l}$ as the input data (see the Supplemental Material [30] for a more detailed description of the procedure). The theoretical results reproduce simultaneously the effective diameters and the peaks of the observed spectra very well (see, e.g., the dashed circles and arrows in Fig. 3).

In Fig. 4 the effective photon potentials obtained for three different excitation powers are compared. A deepening of the well is observed as the power is increased, while the width turns out to be fairly constant, as expected from the invariant laser spot shape. This agreement allows one to conclude that, at least for low excitation powers, the laser-induced potential shape is essentially independent of the laser power (its Gaussian shape and lateral width $\sigma$ remain unaltered), being only the well depth is strongly sensitive to the excitation power. In fact, as can be observed in Fig. 1(d), the photon potential depth shows a supralinear dependence with laser power above $\sim 40 \mathrm{~mW}$. In principle the number of photoexcited carriers should be proportional to the incident power. The observed quadratic dependence might be reflecting an increased absorption at the fixed laser energy, for example due to a GaAs gap reduction induced by a temperature increase.

This latter result points toward the physical origin of the proposed Gaussian photon potential. The potential, schematized in Fig. 1(b), represents the effective local energy of the optical cavity mode of an equivalent planar structure. It is this lateral gradient of the cavity mode energy that leads to light confinement and to new photonic modes (just like a quantum well leads to new confined electronic states defined by, though shifted from, the confinement potential). Similar ideas have been applied to model effective phonon potentials [36]. The resulting modes are redshifted (with respect to the unperturbed planar structure) by the laser excitation, mapping out the shape of the focused laser intensity profile [see, e.g., Fig. 2(c)]. As mentioned in the Introduction, either electronic or thermal phenomena can be at the basis of the observed phenomena. The different phenomena have, however, very different dynamics, typically reflecting picoand nanosecond recombination when photoexcited carriers are responsible [37], and microsecond thermal diffusion times if heating is dominant [38]. To clarify this issue, the dynamics of the photon potential was studied using modulated optical excitation. The modulation was achieved with an acoustooptic modulator, fed with a square-wave generator of tunable frequency and duty cycle.

The top-left panel in Fig. 5 presents photoluminescence spectra as a function of the modulation frequency, for a duty cycle of $20 \%$. From blue to red, the curves correspond to modulation frequencies ranging from $1 \mathrm{MHz}$ to $1 \mathrm{~Hz}$. The figure clearly shows the 3D confined spectra for slow modulation (similar to the $\mathrm{cw}$ experiments shown above), which evolve into the laterally unconfined planar situation with increasing modulation frequency. The top-right panel in Fig. 5 (corresponding to a slightly different position on the sample) displays the photoluminescence spectra variation with duty cycle for a square-wave modulation of $400 \mathrm{kHz}$. This particular frequency was selected because it is where the discrete modes start to be noticed for the minimum duty cycle available (20\%). From blue to red, the curves correspond to duty cycles ranging from $20 \%(0.5 \mu$ s on, $2.0 \mu$ s off $)$ to $80 \%(2.0 \mu \mathrm{s}$ on, $0.5 \mu \mathrm{s}$ off $)$, with steps of $5 \%$. The times involved in the observed dynamics clearly point toward a thermal phenomenon. We note that these results can be related to the "thermal lensing" previously investigated in vertical cavity surface emitting lasers, by which the laser mode lateral distribution is changed depending on the drive power, due to nonuniform heating $[39,40]$.

Having established the thermal origin of the phenomena, we note that two different effects could in principle be responsible for the observed features: a local increase of the refractive index, or a local expansion of the structure. Calculating the spectral position of the optical mode with the transfer matrix method (considering the expansion coefficient and the dependence on temperature of the refractive index of the materials), it turns out that, for a given increase in temperature, the effect of the refractive index change on the spectral position of the optical mode is roughly about eight times larger than that due to the thermal expansion of the lattice. 

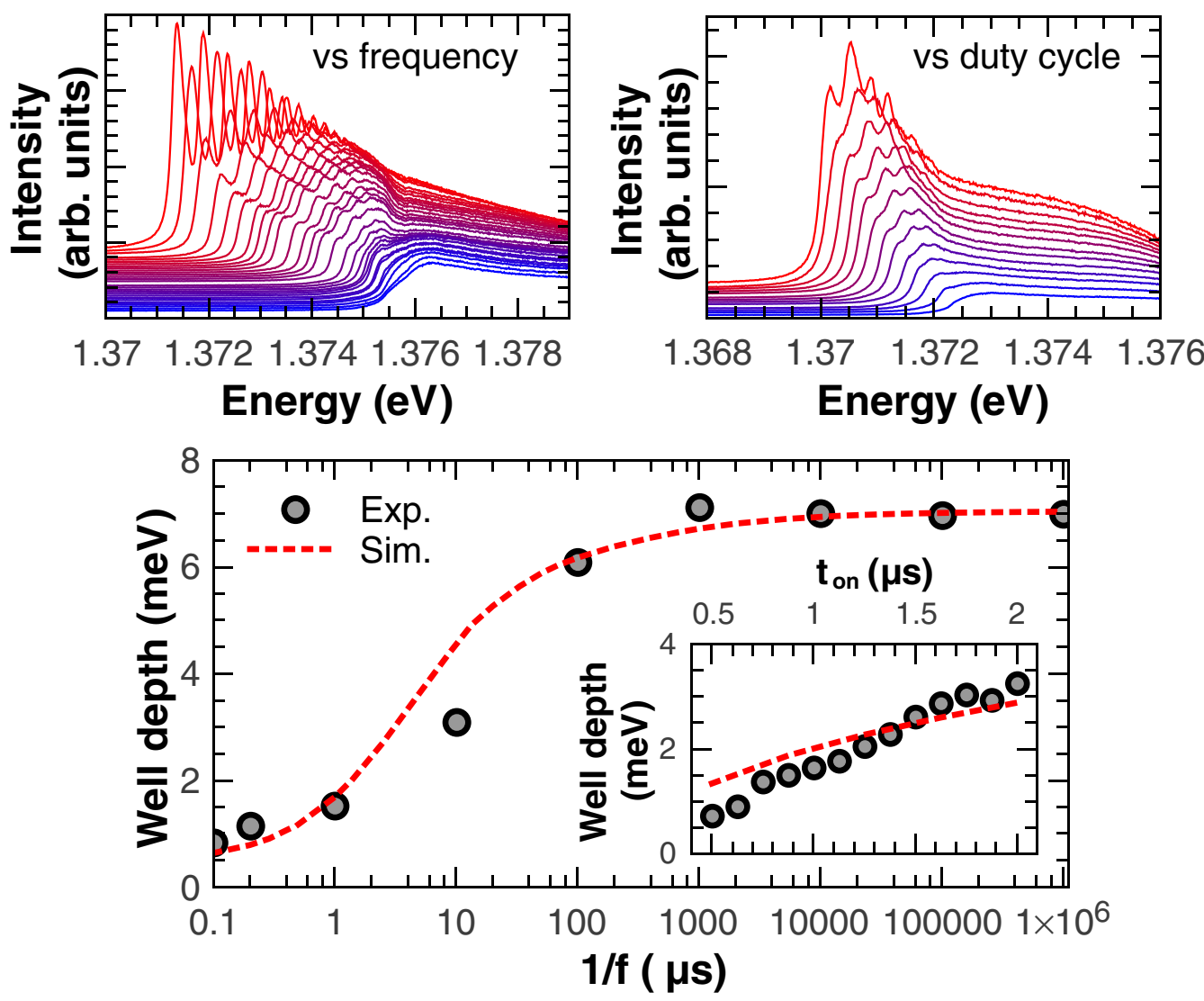

FIG. 5. Top panel, vs frequency: photoluminescence spectra as a function of frequency for a $20 \%$ duty cycle square-wave modulation, with excitation power of $41.3 \mathrm{~mW}$. From blue to red, the curves correspond to frequencies ranging from $1 \mathrm{MHz}$ to $1 \mathrm{~Hz}$. Note that the selected frequencies are not equidistant; e.g., the three most shifted spectra correspond to $1 \mathrm{~Hz}, 1 \mathrm{kHz}$, and $5 \mathrm{kHz}$. vs duty cycle: photoluminescence spectra as a function of the duty cycle for a square-wave modulation of $400 \mathrm{kHz}$ and an excitation power of $33 \mathrm{~mW}$, taken at a slightly different position on the sample. From blue to red, the curves correspond to duty cycles ranging from $20 \%$ to $80 \%$, with steps of $5 \%$. Bottom panel, main frame: optical well depth as a function of the modulation period for an excitation of $47 \mathrm{~mW}$ and a duty cycle of $20 \%$, taken at a slightly different position on the sample. Inset: optical well depth as a function of the duty cycle time. The circles correspond to the experimental results, taken from the curves shown in the top-rightmost panel, while the red dotted curves were obtained from a thermal diffusion model.

Assuming that the change in the refractive index that gives rise to the optical well is directly proportional to the local change in temperature due to the laser excitation, it is possible to describe the physical situation as a heat diffusion problem. A thermal diffusion model was used to obtain the structure's mean temperature during excitation, as a function of the modulation frequency and duty cycle (see the Supplemental Material [30] for a detailed description of the calculations). The thermal diffusivities used in the model were taken from the literature, and are included in the Supplemental Material [30]. Since the exact fraction of the incident laser power that is actually converted into heat is difficult to determine, we used the thermal source maximum value (at the top $\mathrm{Ga}_{0.9} \mathrm{Al}_{0.1} \mathrm{As}$ layer) as the (only) fitting parameter.

In the bottom panel in Fig. 5 we show a comparison between the experimentally observed optical potential well depth (circles), derived from experimental curves as in the top-left figure (though taken at a different power and position on the sample) and the theoretical results (red dashed line) obtained from the thermal diffusion model (maximum heating power of $1.0 \frac{\mathrm{mW}}{\mu \mathrm{m}^{3}}$ ), as a function of the inverse of the modulation frequency $f$. The data in the inset corresponds to the well depth as a function of the duty cycle time, obtained from the measurements shown in the top-right panel, again compared with the theoretical curve derived from the thermal diffusion model (maximum heating power of $0.5 \frac{\mathrm{mW}}{\mu \mathrm{m}^{3}}$ ). In order to estimate the optical potential depth for these measurements, there are two possibilities. If the confined modes are well defined in energy, the method explained before (Bessel modes) could be used; if the modes are somewhat blurred, as occurs for high-frequency modulation, or are too weakly confined to appreciate them, it is still possible to estimate the potential well depth from the envelope function of the emitted photoluminescence. The two methods give similar results, but to be able to compare the high- and low-frequency modulations in Fig. 5, as well as the long and short duty cycles, the second one was used. The agreement between experiment and theory shown in Fig. 5 clearly confirms thermal effects at the origin of the reported light trapping phenomena.

\section{CONCLUSIONS}

In conclusion, we note that equivalent 3D confinement was obtained using a $514 \mathrm{~ns}$ laser, which is strongly absorbed at the first layers of the top DBR, thus only weakly coupling with the rest of the structure. This implies that two-laser 
schemes may be implemented, with one laser used to seed the cavity emission, and the other to dynamically control the 3D light trapping. We note that the GaAs gap strongly depends on temperature above $\sim 100 \mathrm{~K}$ [41]. Below this temperature electronic nonlinearities (which are typically weaker but faster) should be dominant over the observed thermal effects. With both approaches rich photon potentials could be accessible using phase and intensity modulated illumination such as that used in super-resolution microscopy and other photonic applications.

\section{ACKNOWLEDGMENTS}

This work was partially supported by the ANPCyT Grants PICT No. 2012-1661 and No. 2013-2047, the French RENATECH network, and the international Franco-Argentinean Laboratory LIFAN (CNRS-CONICET).
[1] T. Sakaguchi, F. Koyama, and K. Iga, Electron. Lett. 24, 928 (1988).

[2] C. Symonds, G. Lheureux, J. P. Hugonin, J. J. Greffet, J. Laverdant, G. Brucoli, A. Lemaitre, P. Senellart, and J. Bellessa, Nano Lett. 13, 3179 (2013).

[3] B.P. Abbott et al. (LIGO Scientific Collaboration and Virgo Collaboration), Phys. Rev. Lett. 116, 131103 (2016).

[4] J. M. Gérard, D. Barrier, J. Y. Marzin, R. Kuszelewicz, L. Manin, E. Costard, V. Thierry-Mieg, and T. Rivera, Appl. Phys. Lett. 69, 449 (1996).

[5] J. P. Reithmaier, M. Röhner, H. Zull, F. Schäfer, A. Forchel, P. A. Knipp, and T. L. Reinecke, Phys. Rev. Lett. 78, 378 (1997).

[6] A. S. Kuznetsov, P. L. J. Helgers, K. Biermann, and P. V. Santos, Phys. Rev. B 97, 195309 (2018).

[7] E. M. Purcell, Phys. Rev. 69, 681 (1946).

[8] D. Kleppner, Phys. Rev. Lett. 47, 233 (1981).

[9] E. Yablonovitch, Phys. Rev. Lett. 58, 2059 (1987).

[10] R. Balili, V. Hartwell, D. Snoke, L. Pfeiffer, and K. West, Science 316, 1007 (2007).

[11] Y. Akahane, T. Asano, B.-S. Song, and S. Noda, Nature (London) 425, 944 (2003).

[12] J. M. Zajac and W. Langbein, Phys. Rev. B 86, 195401 (2012).

[13] R. I. Kaitouni, O. El Daï, A. Baas, M. Richard, T. Paraiso, P. Lugan, T. Guillet, F. Morier-Genoud, J. D. Ganière, J. L. Staehli, V. Savona, and B. Deveaud, Phys. Rev. B 74, 155311 (2006).

[14] S. L. McCall, A. F. J. Levi, R. E. Slusher, S. J. Pearton, and R. A. Logan, Appl. Phys. Lett. 60, 289 (1992).

[15] V. R. Almeida, C. A. Barrios, R. R. Panepucci, and M. Lipson, Nature (London) 431, 1081 (2004).

[16] S. F. Preble, Q. Xu, B. S. Schmidt, and M. Lipson, Opt. Lett. 30, 2891 (2005).

[17] S. Schmitt-Rink, D. S. Chemla, and D. A. B. Miller, Phys. Rev. B 32, 6601 (1985).

[18] S. Schmitt-Rink, D. Chemla, and D. Miller, Adv. Phys. 38, 89 (1989).

[19] M. Cardona and M. L. W. Thewalt, Rev. Mod. Phys. 77, 1173 (2005).

[20] A. Amo, S. Pigeon, C. Adrados, R. Houdré, E. Giacobino, C. Ciuti, and A. Bramati, Phys. Rev. B 82, 081301(R) (2010).

[21] E. Wertz, L. Ferrier, D. D. Solnyshkov, R. Johne, D. Sanvitto, A. Lemaître, I. Sagnes, R. Grousson, A. V. Kavokin, P. Senellart et al., Nat. Phys. 6, 860 (2010).

[22] G. Tosi, G. Christmann, N. G. Berloff, P. Tsotsis, T. Gao, Z. Hatzopoulos, P. G. Savvidis, and J. J. Baumberg, Nat. Phys. 8, 190 (2012).

[23] A. T. Hammack, M. Griswold, L. V. Butov, L. E. Smallwood, A. L. Ivanov, and A. C. Gossard, Phys. Rev. Lett. 96, 227402 (2006).
[24] G. Roumpos, W. H. Nitsche, S. Höfling, A. Forchel, and Y. Yamamoto, Phys. Rev. Lett. 104, 126403 (2010).

[25] F. Salin and J. Squier, Opt. Lett. 17, 1352 (1992).

[26] D. Bajoni, P. Senellart, A. Lemaître, and J. Bloch, Phys. Rev. B 76, 201305(R) (2007).

[27] M. V. Kurik, Physica Status Solidi A 8, 9 (1971).

[28] J. B. Khurgin, Phys. Rev. B 77, 235206 (2008).

[29] J. B. Khurgin, Appl. Phys. Lett. 104, 221115 (2014).

[30] See Supplemental Material at http://link.aps.org/supplemental/ 10.1103/PhysRevB.99.195308 for details on the experimental setup and the theoretical models used to explain the spatial and spectral distribution of the optically confined modes, which includes Refs. [42-52].

[31] M. Aichinger, S. A. Chin, E. Krotscheck, and E. Räsänen, Phys. Rev. B 73, 195310 (2006).

[32] R. Houdré, C. Weisbuch, R. P. Stanley, U. Oesterle, P. Pellandini, and M. Ilegems, Phys. Rev. Lett. 73, 2043 (1994).

[33] C. Reinhardt, R. Brückner, J. Haase, M. Sudzius, S. I. Hintschich, H. Fröb, V. G. Lyssenko, and K. Leo, Appl. Phys. Lett. 100, 103306 (2012).

[34] T. Gutbrod, M. Bayer, A. Forchel, P. A. Knipp, T. L. Reinecke, A. Tartakovskii, V. D. Kulakovskii, N. A. Gippius, and S. G. Tikhodeev, Phys. Rev. B 59, 2223 (1999).

[35] O. Gazzano, S. M. de Vasconcellos, K. Gauthron, C. Symonds, J. Bloch, P. Voisin, J. Bellessa, A. Lemaître, and P. Senellart, Phys. Rev. Lett. 107, 247402 (2011).

[36] N. D. Lanzillotti-Kimura, A. Fainstein, C. A. Balseiro, and B. Jusserand, Phys. Rev. B 75, 024301 (2007).

[37] S. Anguiano, A. E. Bruchhausen, I. Favero, I. Sagnes, A. Lemaître, N. D. Lanzillotti-Kimura, and A. Fainstein, Phys. Rev. A 98, 013816 (2018).

[38] M. Soltanolkotabi, G. L. Bennis, and R. Gupta, J. Appl. Phys. 85, 794 (1999).

[39] M. Brunner, K. Gulden, R. Hövel, M. Moser, and M. Ilegems, Appl. Phys. Lett. 76, 7 (2000).

[40] R. Amatya, D. Luerssen, M. Farzaneh, and J. A. Hudgings, in Conference on Lasers and Electro-Optics/Quantum Electronics and Laser Science Conference and Photonic Applications Systems Technologies, Technical Digest (CD) (Optical Society of America, 2006), paper JWB17.

[41] C. Kim, P. Lautenschlager, and M. Cardona, Solid State Commun. 59, 797 (1986).

[42] D. B. Williams and C. B. Carter, Transmission Electron Microscopy (Springer, New York, 2009).

[43] P. Y. Yu and M. Cardona, Fundamentals of Semiconductors: Physics and Materials Properties, 3rd ed. (Springer, Berlin, 2001).

[44] J. Crank, The Mathematics of Diffusion (Clarendon, Oxford, 1975). 
[45] R. Ghez, A Primer of Diffusion Problems (Wiley, New York, 1988).

[46] L. C. Andrews, Special Functions of Mathematics for Engineers (SPIE, Washington, 1998), p. 110.

[47] R. O. Carlson, G. A. Slack, and S. J. Silverman, J. Appl. Phys. 36, 505 (1965).
[48] J. S. Blakemore, J. Appl. Phys. 53, R123 (1982).

[49] M. A. Afromowitz, J. Appl. Phys. 44, 1292 (1973).

[50] B. D. Lichter and P. Sommelet, Trans. Metall. Soc. AIME 245, 1021 (1969).

[51] J. Shen and A. Mandelis, Rev. Sci. Instrum. 66, 4999 (1995).

[52] C. Wang and A. Mandelis, Rev. Sci. Instrum. 70, 2372 (1999). 